\title{
Radiation of a Circular Arc Source in a Limited Angle for Non-uniform Conformal Arrays
}

\author{
G. Leone, F. Munno, and R. Pierri
}

\begin{abstract}
An inverse problem approach is adopted to investigate the role of a limited observation domain in conformal source radiation both in far and near zone. The spectral decomposition of the relevant operator drives the investigation, especially by the discussion of the singular values behavior and the Point Spread Function (PSF). For a circular source geometry, accurate closed form evaluations of the PSF allow to establish its angular variant behavior for limited observation angular domains. This leads to introduce a numerical procedure, based on the widths of the PSF main lobes, able to define an optimal source discretization, that is the spacing of the array elements whose radiated field has the same number of degrees of freedom of the continuous conformal source. A non-uniform spacing is derived and the performances of the corresponding conformal arrays are compared with the uniform case, the number of elements being equal. Numerical results about pattern synthesis and array diagnostics of faulty elements support the improvement achieved by the approach when the same number of array elements are located in a non-uniform pattern.
\end{abstract}

Index Terms - Conformal antennas, Electromagnetic scattering inverse problems, Inverse source problem, Number of Degrees of Freedom, Singular Value Decomposition.

\section{INTRODUCTION}

$\mathrm{T}$ HE radiation properties of antennas of general shape depend on both the source geometry and the angular domain of interest. A planar antenna can radiate a focusing broadside beam, while scanning its maximum leads to beam broadening. Instead, a spherical source can radiate identical beams, but occupies a large volume. In principle, intermediate geometries may provide a good trade-off between electrical and geometrical requirements. The radiation properties depend also on the source discretization, that is the spacing between individual elements in an array antenna. An optimal configuration requires the least number of sources to minimize the feeding network complexity and the mutual coupling influence. Conformal arrays are commonly designed with equispaced elements, a criterion derived from the planar ones in order to suppress radiation via grating lobes. However, in some circumstances the array element positions have been arranged in a non-uniform way to enhance the array performances. The elements' positions have been determined by various approaches, mostly relating to the minimization of

This work was supported by the European Union and the Italian Ministry of University and Research funding through "Programma Operativo Nazionale Ricerca e Innovazione 2014-2020”. some cost functional. For instance in [1] a combination of global and local minimization algorithms are employed to design the positions of spherical array elements while in [2] a stochastic minimization procedure is adopted for a multiobjective optimization problem and applied to a limited number of array elements, as in [3] by the genetic algorithm. Also, in [4] a local minimization under least square condition is performed for non-uniform array by representation of the far field under orthogonal basis. [5] resorts to the compressive sensing approach to introduce a positioning synthesis algorithm.

Due to the complexity of the problem, all procedures lead to ad hoc solutions, without taking into account the peculiar radiation properties of the source according to its geometry.

On the other hand, the source optimal discretization issue is an important problem whenever, in testing and diagnostics, the near zone field is to be probed and reliable results are required also when only a limited observation domain is available.

A unified strategy to deal with these points may be provided by adopting an inverse problem approach [6,7] where the spectral decomposition of the relevant operator is investigated.

In particular, the ill-conditioning $[8,9]$ of the relevant integral operator implies that only a finite number of source current functions can be correctly reconstructed in presence of uncertainties on data, otherwise small noise on data can provide a large error on the reconstructed current. As a matter of fact, this can provide limitations on the number of array elements that can be reliably diagnosed in an array antenna. On the other hand, the above regularization issue narrows the set of fields that can be radiated by the source, too. In far zone, this can reduce the set of achievable radiation patterns and impacts on the antenna synthesis problem.

For these reasons it is important to evaluate the Number of Degrees of Freedom (NDF) [10-12] of the source current in connection to its geometry, as the (finite) number (i.e. the dimension of the subspace) of source current functions that can be stably reconstructed [12].

In addition, this number is also related to the achievable resolution [13], i.e. to the capability of the solution algorithm to reconstruct two close point-like sources. This is associated to the Point Spread Function (PSF) whose properties are fixed by

Giovanni Leone, Fortuna Munno and Rocco Pierri are with the Dipartimento di Ingegneria, Università della Campania Luigi Vanvitelli, Aversa, I-81031, Italy. (e-mail:_ giovanni.leone@unicampania.it, fortuna.munno@unicampania.it,rocco.pierri@unicampania.it) 
the NDF. It is clear that a detailed knowledge about the NDF and, hence, the achievable resolution is of primary importance in these kinds of problems.

In [6], for the first time, we proposed to apply the Singular Value Decomposition (SVD) to the analysis of the radiation operator connecting a source with non rectilinear geometry to the far field. The final goal is to provide a general way of comparing their radiation properties in connection with their geometries by examination of the numerical results of the SVD of the relevant operators. However, the main results about the Singular Values' (SVs) behavior and NDF are only numerical and only a qualitative comparison of some different source geometries is performed.

In $[7,14]$ a circumference conformal geometry is fully examined by the SVD approach, with the effort of providing closed form evaluations and/or estimation of the NDF. First, for different full angle circles, closed form SVDs are recalled or derived and discussed. Next, an arc of circumference is considered, and asymptotic arguments are introduced and numerically validated to provide an estimate of both the NDF and the Singular Functions (SFs) behavior. To this end, the main result is concerned with the role of the source angle, or, rather, its electrical length, in determining the NDF and, thus, the dimension of the subspace of realizable far field patterns. Finally, the role of the NDF is sketched with a simple numerical example of the synthesis of a focusing beam pointing at different directions. In [15] the approach has been applied to other conformal conic curves, while in [16] it has led to define an optimal array geometry for wide angle coverage

In this paper we are interested in examining the role of a finite observation domain on the spectral analysis of the radiation operator both in far and in the near zone and discuss the consequences on optimal spacing of the elements of a conformal array. The proposed analysis, which is formulated in Section II, is based on the above mentioned two parameters arising in the regularized solution of linear inverse problems: the NDF, which is connected to the SVs behaviour, and the PSF, which is connected to the SFs. Since for a circumference geometry some analytical result for the SVD of the relevant operators can be found, our discussion is developed for this case. In fact, in Section III, closed form expressions of the SVs can be established when the observation domain is the round angle, also in presence of a weight factor to be introduced to improve the accuracy of the evaluation of the PSF. The role of the introduced weight function in providing a flatter behaviour of the SVs is emphasized, both in the far and then ear zone. In addition, the same behaviour is numerically verified in the case of a limited angular observation domain.

The next step of the analysis, discussed in Section IV, concerns with the PSF evaluation. A closed form approximate form is derived and its accuracy verified. For large observation domains an angularly invariant behaviour is predicted, that is the invariance of its main beam with respect to the maximum direction, whereas for smaller observation domain things change markedly.

The angularly variant behaviour of the PSF is exploited in Section $\mathrm{V}$ to define a discretized source providing the same
NDF of the continuous one. A procedure is introduced leading to a non-uniform step of the elementary discretized sources. In this way their number is optimized with respect to the uniform counterpart, so that it can be reduced to a minimum the NDF being equal.

Sections VI and VII are devoted to show numerical results about the applications of the previous results to some problems of interest in antenna engineering. In particular, the role of an optimal non-uniform discretization is emphasized in array synthesis and diagnostics in comparison with a uniform one. Conclusions end the paper.

\section{Problem Geometry}

We suppose that the source is an $y$-oriented surface current density function $J(\phi)$ supported over a circumference arc of radius $R$ laying in the $x-z$ plane, with $\phi$ being the angular variable spanning the angular domain $[-\alpha, \alpha]$ of the source. The radiated electric field $E(\theta)$ is observed over a circumference arc in the angular variable $\theta$, spanning the observation domain $[-\gamma, \gamma]$ (see Fig. 1).

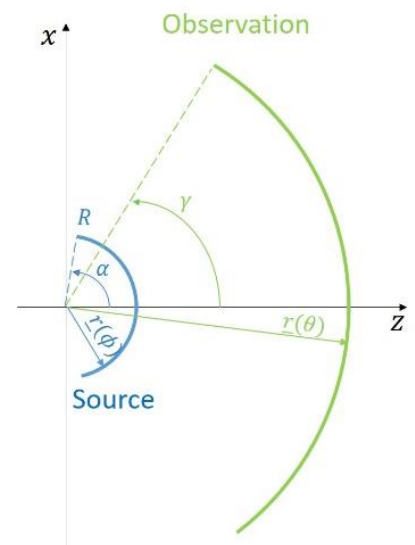

Fig. 1 Considered geometry.

By introducing the subscript $i \in\{F, N\}$ for identifying the case we are referring to, whether to a far $(F)$ or a near $(N)$ observation zone, the electric field, except for inessential factors, writes as

$E_{i}(\theta)=R \int_{-\alpha}^{\alpha} J(\phi) G_{i}\left(R, \phi, R_{o}, \theta\right) d \phi=A_{i} J$,

where $\beta=2 \pi / \lambda$ is the wavenumber, $\lambda$ is the wavelength and $R_{o}$ is the radius of the observation arc in near zone. The $G_{i}(\cdot)$ function represents the $2 \mathrm{D}$ Green function particularized to each observation zone when the background is a homogeneous one, namely,

$G_{i}\left(R, \phi, R_{o}, \theta\right)=\left\{\begin{array}{cc}e^{j \beta R \cos (\theta-\phi)} & \text { for } i=F \\ H_{0}^{(2)}(\beta|\underline{r}(\theta)-\underline{r}(\phi)|) & \text { for } i=N\end{array}\right.$,

where $\underline{r}(\theta)$ is the position vector pointing at the observation point at $\theta$ angle, $\underline{r}(\phi)$ is the position vector of the source point at $\phi$ angle, and $H_{0}^{(2)}(\cdot)$ is the second kind Hankel function of 
zeroth order. Equation (1) defines the radiation operator $A_{i}$ between source and observation domain, with $J$ and $E_{i}$ belonging to $L_{[-\alpha, \alpha]}^{2}$ and $L_{[-\gamma, \gamma]}^{2}$, respectively, that is, the set of square integrable functions supported over the interval specified by the subscript.

In the following analysis, we refer to the SVD [9] of the relevant operator. It consists of the left singular functions $u_{i v}$, the singular values $\sigma_{i v}$ and the right singular functions $v_{i v}$. In particular, the $\left\{u_{i v}\right\}$ and $\left\{v_{i v}\right\}$ functions correspond to an orthonormal basis of the operator range and operator domain, respectively. Since for a compact operator as in (1), with a kernel function behaving like an entire function of exponential type, the singular values decay exponentially fast, the NDF of the source can be defined as the number of "significant" singular values [12]. It provides the dimension of the subspace of fields that can be represented with an assigned accuracy and identifies the dimension of the subspace of sources that can be correctly reconstructed.

\section{Singular VALUES BehaVior}

In order to consider a more general operator we introduce an appropriate weight function $h(\theta-\phi)$ inside the integral operators (1). This can be associated to an element factor of the current, identical for each source element, except for a rotation. For a circumference source and an element factor maximum at the zero argument, it provides a directive behavior pointing at the normal direction of each source element. For an array source it provides exactly the element factor of the antenna. From now on, hence, the radiation operators to be considered can be rewritten as

$E_{i}(\theta)=R \int_{-\alpha}^{\alpha} J(\phi) h(\theta-\phi) G_{i}\left(R, \phi, R_{o}, \theta\right) d \phi=A_{i} J$

In this paper we have chosen to consider the weight functions

$h(\theta-\phi)=(1+\cos (\theta-\phi))^{m}, m=0,1,2, \ldots$

As $m$ increases, (4) provides a more directive single lobe pattern, while for $m=0$ the effect of the weight function is cancelled and the standard radiation operators are recovered.

When $\alpha=\gamma=\pi$, i.e. the case of a circumference source observed on a full angle, a closed form analytical evaluation of the eigenvalue decompositions of (3) are provided in Appendix A. In particular, the analytical results of the eigenvalues $\lambda_{\text {in }}$ both in the far

$\lambda_{F n}=2^{-m+1} \pi R \sum_{k=0}^{2 m}\left(\begin{array}{c}2 m \\ k\end{array}\right) j^{n+k-m} J_{n+k-m}(\beta R)$

and the near zone

$\lambda_{N n}=2^{-m+1} \pi R \sum_{k=0}^{2 m}\left(\begin{array}{c}2 m \\ k\end{array}\right) J_{n+k-m}(\beta R) H_{n+k-m}^{(2)}\left(\beta R_{o}\right)$

are available.
In Appendix $\mathrm{A}$ it is also shown how the above expressions can be rewritten as a combination of a reduced number of Bessel functions (for the $n$-th eigenvalue, the Bessel/Hankel functions of $n$-order and their derivatives). In particular, for the very first values of $m$ it is obtained:

- for $m=0$

$\lambda_{F n}=2 \pi R j^{n} J_{n}(\beta R)$,

$\lambda_{N n}=2 \pi R J_{n}(\beta R) H_{n}^{(2)}\left(\beta R_{o}\right) ;$

- for $m=1$

$$
\begin{gathered}
\lambda_{F n}=2 \pi R j^{n}\left[J_{n}(\beta R)-j J_{n}^{\prime}(\beta R)\right], \\
\lambda_{N n}=2 \pi R\left[J_{n}^{\prime}(\beta R) H_{n}^{(2) \prime}\left(\beta R_{o}\right)+\right. \\
\left.\left(\frac{n^{2}}{\beta^{2} R R_{o}}+1\right) J_{n}(\beta R) H_{n}^{(2)}\left(\beta R_{o}\right)\right]
\end{gathered}
$$

In Fig 2 the $\left|\lambda_{\text {in }}\right|$ of (5) and (6) are plotted in descending order according to the $v$ index, for different $m$. First of all, we point out that all curves decay exponentially for indices $v>$ $N_{N D F}=2[\beta R]+1$ as to be expected since the NDF of any circumference source is $N_{N D F}$ [7] independently from the source current. In addition, they exhibit a flattening for any $m \neq$ 0 , so leading to a more pronounced step-like behavior beyond the NDF value. In particular, the more $m$ increases and the more the behavior flattens in the case of the near zone, while for the far zone the maximum observed flattening is for $m=2$.
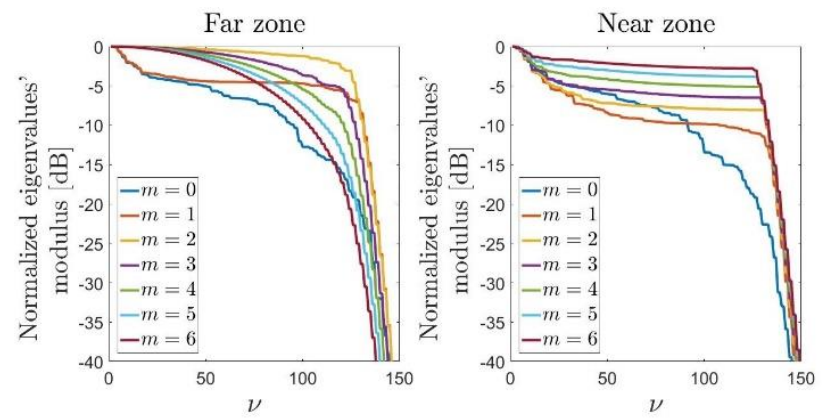

Fig. 2 Normalized eigenvalues' modulus $\left|\lambda_{F n}\right|$ and $\left|\lambda_{N n}\right|$ by (5) and (6) respectively, sorted in decreasing order, of $\mathcal{A}_{\mathrm{F}}$ (left panel) and $\mathcal{A}_{\mathrm{N}}\left(\mathrm{R}_{\mathrm{o}}=15 \lambda\right)$ (right panel) for a circumference source of radius $\mathrm{R}=10 \lambda$, for different $\mathrm{m}$ values of the weight (4).

The flattening effect of a fixed $m$ depends on the observation radius $R_{o}$ also. This is shown in the example of Fig. 3, where the curves corresponding to $m=2, m=3$ and $m=5$ are plotted varying $R_{o}$ for the same configuration of Fig. 2. In fact, for $m>2$, the eigenvalues curves become smoother as $R_{o}$ increases. On the contrary, for $m=2$ it behaves flatter as $R_{o}$ increases, while for $m=0$ it is always a smooth function of the index. Therefore, the choice $m=2$ allows to achieve a flatter behavior at every observation distance from the source. This result can be exploited when the flat behavior of the singular functions (more than their dynamic range) plays an important role in approximating a function of interest of the present 
discussion, as it is shown in next Section as far as the PSF is concerned.
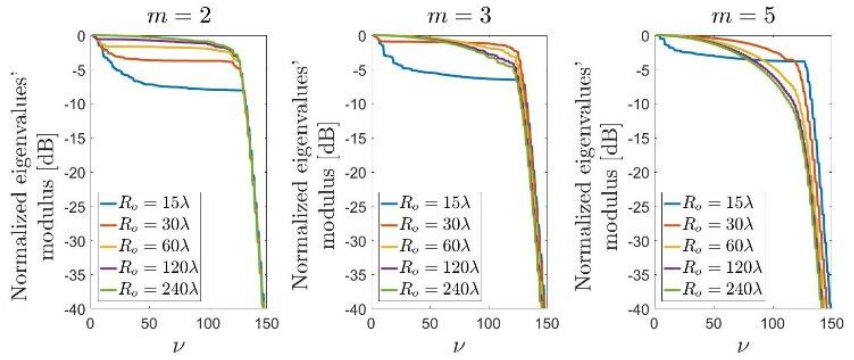

Fig. 3 Normalized eigenvalues' modulus $\left|\lambda_{N n}\right|$ by (6), sorted in decreasing order, of $\mathcal{A}_{N}$ for a circumference source of radius $R=10 \lambda$, for different $\mathrm{m}$ values in the weight and different radii $R_{o}$.

In the more general case of limited angular intervals for both source and observation, an analytic expression of the singular values is no longer available, and it is necessary to evaluate them numerically by densely discretizing both source and observation domains. However, the above analytical results can be usefully employed to predict the general behavior of the SVs. In fact, Figs. 4 and 5 show that also for limited source and observation the introduction of the weight function (4) allows to obtain a flatter behavior of the SVs before their common exponential decay. It refers to the case of a semi-circumference observed on a $3 \pi / 4$ angle. Since similar numerical results have been obtained for other source and observation geometries, we can confirm again that an NDF estimation found for $m=0$ still works for $m \neq 0$ and that the effect of the introduced weight function (4) consists only in flattening the behavior of the SVs.

Since for every source and observation geometries the flatter behavior of the SVs is achieved for $m=2$ in the weight function (4), this choice is pursued in all numerical examples to follow.
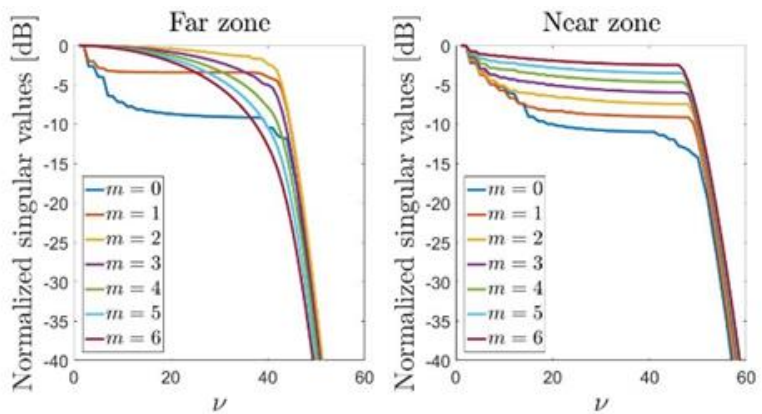

Fig. 4 Numerically computed normalized singular values of $\mathcal{A}_{F}$ (left panel) and $\mathcal{A}_{N}$ (right panel, $R_{o}=15 \lambda$ ), for an arc of circumference source of radius $R=$ $10 \lambda, \alpha=\pi / 2$ and $\gamma=3 \pi / 4$.

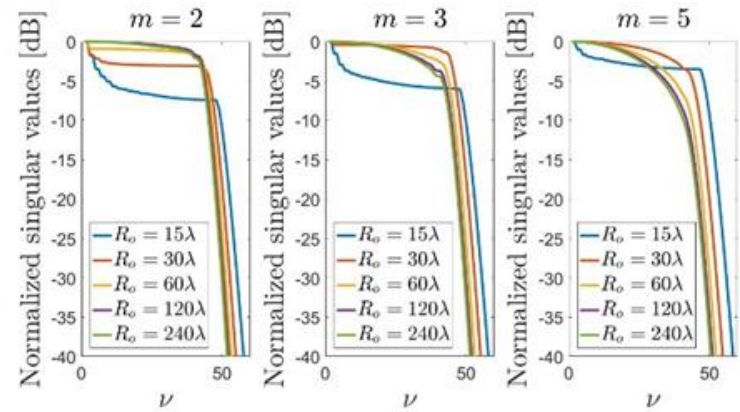

Fig. 5 Numerically computed normalized singular values of $\mathcal{A}_{\mathrm{N}}$ of a circumference arc source with radius $R=10 \lambda$ and $\alpha=\pi / 2$ observed on a circumference arc with $\gamma=3 \pi / 8$ and varying radius $R_{0}$.

\section{PSFs EVALUATION}

In this section, we are concerned with an approximate analytical expression of the PSF both in the source domain and in the observation domain. The PSF, by definition, represents the impulsive response of a system formed from the cascade of the regularized inverse operator and the direct operator, leading, for instance, in the source domain to

$\operatorname{PSF}_{i}\left(\phi, \phi_{0}\right)=\mathcal{A}_{i}^{-1} \mathcal{A}_{i} \delta\left(\phi^{\prime}-\phi_{0}\right)$,

where $\delta(\cdot)$ represents the Dirac impulsive function centered at the source domain point $\phi_{0}$.

The amplitude of the main lobe of the PSF, in particular, is linked to the resolution issue in inverse source problems, that is the capability of the inversion scheme to distinguish reliably two close point-like sources. This affects both the problems of array antenna synthesis and diagnostics. In fact, for instance, by adjusting the spacing of the array elements according to the amplitude of the main lobe of the PSF, we may be able to achieve the best possible performances by the least number of radiating elements.

We adopt the Truncated SVD algorithm (TSVD) [9] to perform the regularized inversion of the relevant operators to be discussed. It consists in retaining in the singular function expansion of the generalized inverse solution only those terms corresponding to singular values greater than a certain threshold value. Such a value is chosen in correspondence of the start of their exponential decay, that is the NDF.

Accordingly, from (11) it follows

$P S F_{i}\left(\phi, \phi_{0}\right)=\sum_{v=1}^{N D F} v_{i v}(\phi) v_{i v}^{*}\left(\phi_{0}\right)$,

so that it involves the right singular functions of operators (3) which, unfortunately, are only analytically known for particular geometrical configurations, as in the case of a source supported on a circumference observed on a full angle (Appendix A). For those cases where (12) is not easily calculable, a different strategy may be used involving an approximation of (12).

To this end we consider the adjoint operator $\mathcal{A}_{i}^{\dagger}$ corresponding to the radiation operator $\mathcal{A}_{i}$ which is defined by the 
$<\mathcal{A}_{i} J, E_{i\rangle_{[-\pi, \pi]}^{2}}=<J, \mathcal{A}_{i}^{\dagger} E_{i}>_{L_{[-\alpha, \alpha]}^{2}}$,

where $\langle\cdot$,$\rangle stands for the scalar product within the$ appropriate space. Then a good approximation of (12) may be achieved by using the adjoint operator as a regularized inverse, namely by reformulating (11) as

$\widetilde{P S F_{i}}\left(\phi, \phi_{0}\right)=\mathcal{A}_{i}^{\dagger} \mathcal{A}_{i} \delta\left(\phi^{\prime}-\phi_{0}\right)$.

By expressing (14) in terms of singular functions, one gets

$\widetilde{P S F_{i}}\left(\phi, \phi_{0}\right)=\sum_{v=1}^{N D F} \sigma_{v}^{2} v_{v}(\phi) v_{v}^{*}\left(\phi_{0}\right)$

where, the same singular functions as in (12) are now weighted by the corresponding singular values. The two expressions (12) and (15), then, are the more similar the more the significant singular values maintain a flat behavior. So the analysis of the previous Section can be helpful to provide a proper choice of $m$ in the weight function of the relevant operators (3) so that the approximate PSF deviates from the exact one in the least.

It is possible to demonstrate (see Appendix B) that (14) leads to the following explicit expression of the PSF in far zone

$$
\begin{gathered}
\widetilde{\operatorname{PSF}_{F}}\left(\phi, \phi_{0}\right)=\gamma R^{2} 2^{-2 m+1} e^{-j m\left(\phi+\phi_{0}\right)} . \\
\sum_{n=-\infty}^{+\infty} J_{n}\left(2 \beta R \sin \left(\frac{\phi_{0}-\phi}{2}\right)\right) e^{-j n \frac{\phi+\phi_{0}}{2}} \sum_{k=0}^{2 m}\left(\begin{array}{c}
2 m \\
k
\end{array}\right), \\
\cdot e^{j k \phi} \sum_{s=0}^{2 m}\left(\begin{array}{c}
2 m \\
s
\end{array}\right) e^{j s \phi_{0}} \operatorname{sinc}[(n+2 m-k-s) \gamma]
\end{gathered}
$$

that for $m=0$ reduces to

$$
\begin{aligned}
\widetilde{P S F_{F}}\left(\phi, \phi_{0}\right)= & 2 \gamma R^{2} \sum_{n=-\infty}^{+\infty} J_{n}\left(2 \beta R \sin \left(\frac{\phi_{0}-\phi}{2}\right)\right) . \\
& \cdot e^{-j n \frac{\phi+\phi_{0}}{2}} \operatorname{sinc}(n \gamma)
\end{aligned}
$$

By similar procedures, for the near zone the approximate PSF is given by

$$
\begin{gathered}
\widetilde{P S F_{N}}\left(\phi, \phi_{0}\right)=\gamma R^{2} 2^{-2 m+1} e^{-j m\left(\phi+\phi_{0}\right)} \sum_{p=-\infty}^{+\infty} J_{p}(\beta R) \cdot \\
\cdot H_{p}^{(2) *}\left(\beta R_{o}\right) e^{j p \phi} \sum_{l=-\infty}^{+\infty} J_{l}(\beta R) H_{l}^{(2)}\left(\beta R_{o}\right) e^{-j l \phi_{0}} \sum_{k=0}^{2 m}\left(\begin{array}{c}
2 m \\
k
\end{array}\right) \\
\cdot e^{j k \phi} \sum_{s=0}^{2 m}\left(\begin{array}{c}
2 m \\
s
\end{array}\right) e^{j s \phi_{0}} \operatorname{sinc}[(2 m-p+l-k-s) \gamma]
\end{gathered}
$$

that for $m=0$ becomes

$$
\begin{gathered}
\widetilde{P S F_{N}}\left(\phi, \phi_{0}\right)=2 \gamma R^{2} \sum_{p=-\infty}^{+\infty} J_{p}(\beta R) H_{p}^{(2) *}\left(\beta R_{o}\right) \cdot \\
\cdot \sum_{l=-\infty}^{+\infty} J_{l}(\beta R) H_{l}^{(2)}\left(\beta R_{o}\right) e^{j\left(p \phi-l \phi_{0}\right)} \operatorname{sinc}[(l-p) \gamma]
\end{gathered}
$$

In order to check, that the presence of the weight term, improves the approximate PSF effectively, we compare the numerically PSFs obtained from (12) with the closed form expressions of the approximate ones for the cases $m=0$ and $m=2$. From Figs. 6 and 7 we can immediately appreciate that the PSF is generally spatially variant along the source domain, implying a non-uniform resolution too.

On the other hand, when $\gamma=\pi$, both $\widetilde{P S F_{l}}$ depend only on the $\phi_{0}-\phi$ difference, which means that they are angularly invariant, so implying an uniform resolution in point-like source reconstructions. This property holds approximately also when $\gamma$ is large; since in this paper we are interested in examining the effect of a limited observation domain, hereafter we consider mainly examples where $\alpha>\gamma$, so enhancing the expected angularly variant behavior of the relevant PSFs.

Furthermore, we see that the choice $m=2$ (which ensures a flattening of the singular values both for the near and the far zones, as discussed in the previous Section) the PSFs (16) and (18) follow better the behavior of the exact PSFs than in the case $m=0$. This result accounts for the need of the deep investigation of the role of the weight function in the operators of interest. In all circumstances a fairly good agreement is obtained especially in the main lobe regions. Therefore, the approximate PSF can be fruitfully employed in the solution of inverse problems founded on its knowledge, as it is shown in next Section.
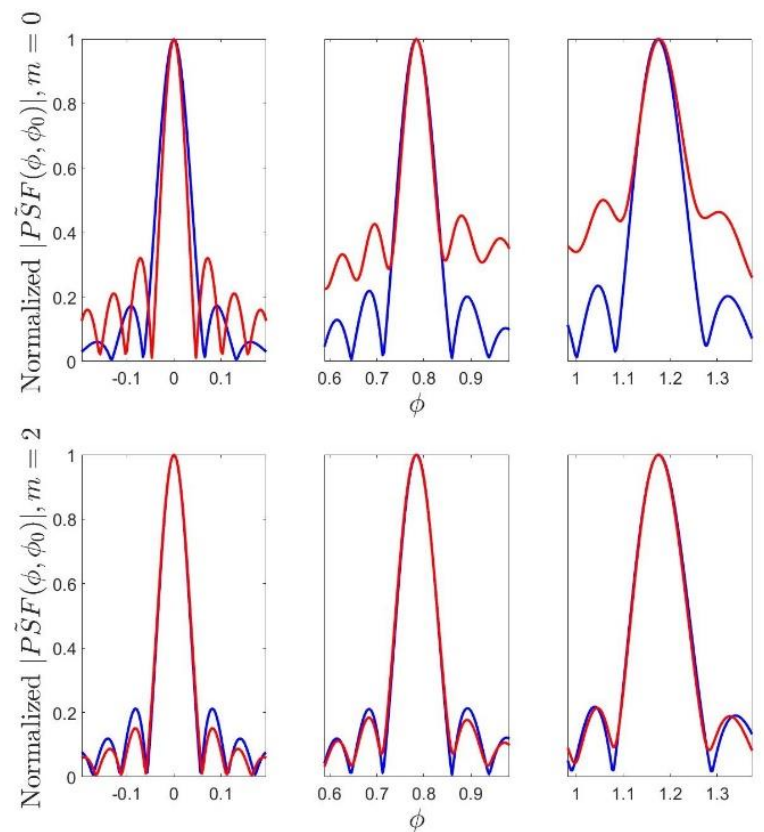

Fig. 6 Normalized amplitude of the $\widetilde{\mathrm{PSF}}_{\mathrm{F}}$ (red line) and the numerically computed PSFF (12) (blue line) for an arc of circumference with $\mathrm{R}=10 \lambda$ and $\alpha=\pi / 2$ observed in far zone for $\gamma=3 \pi / 8$. The upper panel refers to the case for $m=0$, whereas the lower panel is for $m=2$. 

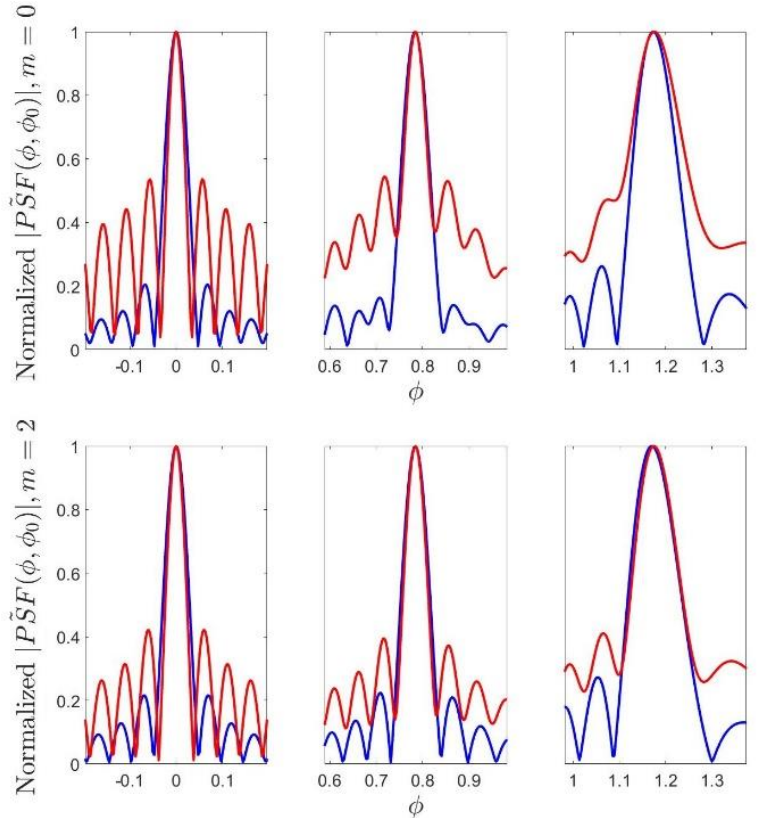

Fig. 7 Normalized amplitude of $\widetilde{\mathrm{PSF}_{\mathrm{N}}}$ (red line) and the numerically computed PSFN (12) (blue line) for an arc of circumference with $\mathrm{R}=10 \lambda$ and $\alpha=\pi / 2$ observed in near zone for $\mathrm{R}_{\mathrm{o}}=15 \lambda$ and $\gamma=3 \pi / 8$. The upper panel refers to the case $m=0$, whereas the lower panel is for $m=2$.

From Figs. 6 and 7, a markedly angularly variant behavior of the PSFs is apparent as also predicted by the analytical functions (16) and (18). However, when $\gamma$ increases, the relevance of the factors depending on the summation $\phi+\phi_{0}$ reduces and a nearly invariant behavior occurs. Figs. 8 and 9 displays numerically this result as $\gamma>\alpha$.
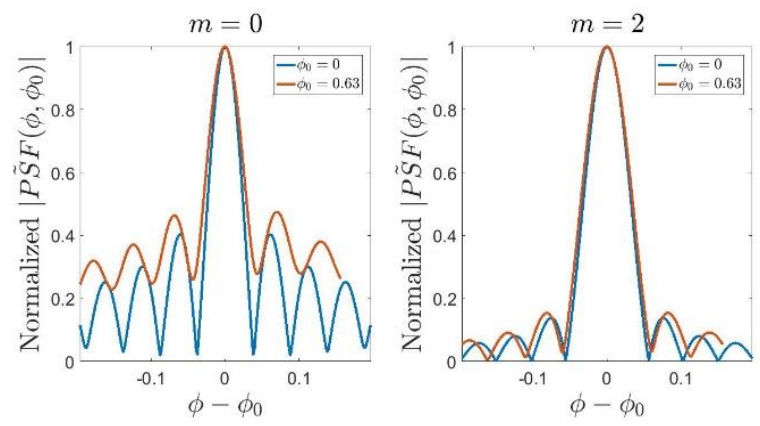

Fig. 8 Normalized amplitude of the $\widetilde{\mathrm{PSF}_{\mathrm{F}}}$ for $\phi_{0}=0$ (blue line) and $\phi_{0}=0.63$ (red line) for an arc of circumference with $\mathrm{R}=10 \lambda$ and $\alpha=\pi / 4$ observed in far zone for $\gamma=\pi / 2$. The left panel refers to the case for $m=0$, whereas the right panel is for $\mathrm{m}=2$.
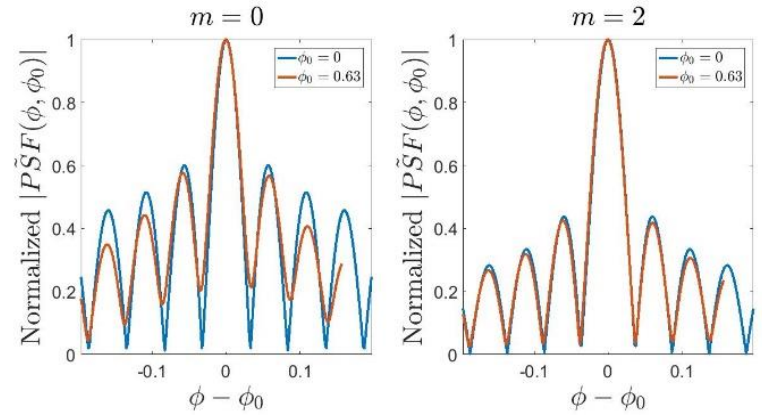

Fig. 9 Normalized amplitude of the $\widetilde{P S F_{N}}$ for $\phi_{0}=0$ (blue line) and $\phi_{0}=$ 0.63 (red line) for an arc of circumference with $R=10 \lambda$ and $\alpha=\pi / 4$ observed in near zone for $\gamma=\pi / 2$ and $R_{o}=15 \lambda$. The left panel refers to the case for $\mathrm{m}=0$, whereas the right panel is for $m=2$.

\section{DisCRETE SOURCES}

The analytical expressions of the PSFs found in the previous Section are not only important to determine the intrinsic resolution limits of the inversion algorithm according to the source and the observation domain geometrical width, but they may be usefully employed to devise a scheme either for an efficient discretization of the source or to adequately sample the radiated field.

In fact, for a prefixed geometry of the source support, an interesting question that can be raised concerns with deducing the positioning and the minimum number of point-like sources able to guarantee the same performances of the corresponding continuous source. At the same time, it would be very useful to investigate whether an efficient way (in terms of the number of measurement points) to sample the radiated field for the purpose of source reconstruction and diagnostics exists, without impairing their accuracy. To this end, the NDF of the relevant radiation operator is the fundamental information to be considered, as the maximum dimension of the set of current functions that can be reliably reconstructed in presence of uncertainties on data. So we assume it as the reference parameter and suppose also that it may provide the maximum number of point-like sources that can be reconstructed reliably. However, the spacing between these sources is not provided by the SVD evaluation of the operators (3) and there is no guarantee that a uniform one might achieve the optimal results, that is, the minimum number of point-like sources capable to radiate a set of fields whose dimension is just the NDF of the corresponding continuous current source.

\section{A. Model Operator}

Now we consider an array of $N A$ point-like elements spaced at $\Delta \phi_{l}^{k}, l=1, \ldots, N A$ step as source, where the superscript $k \in$ $\{N U, U\}$ refers to either a non-uniform or an uniform angular step. The discrete counterpart of the radiation operators (3) are to be defined in terms of two semi-discrete mappings $\tilde{\mathcal{A}}_{i}, i=$ $\{F, N\}$ transforming vectors into functions. Hence, (3) leads to the operators

$\tilde{E}_{i}(\theta)=\sum_{l=1}^{N A} c_{l} I_{l} h\left(\theta-\phi_{l}\right) G_{i}\left(R, \phi_{l}^{k}, R_{o}, \theta\right)=\tilde{A}_{i} \underline{I}$,

mapping the vector $\underline{I} \in \mathbb{C}^{N A}$ into the function $\tilde{E}_{F} \in L_{[-\gamma, \gamma]}^{2}$, where $c_{l}=R \Delta \phi_{l}^{k}$. Each component of the $\underline{I}$ vector represents the $I_{l}$ array excitation coefficient corresponding to the $l$-th element of the $k$ configuration.

The array element positions are defined by the angles

$\phi_{l}^{k}=-\alpha+\Delta \phi_{l}^{k}$ for $l=1, \ldots, N A$

belonging to the arc of circumference with radius $R$ within the angular interval $\phi \in(-\alpha, \alpha)$. Therefore the relevant SVD of (20) consists in the triple $\left\{\tilde{\sigma}_{i v}, \tilde{u}_{i v}(\theta), \underline{\tilde{v}}_{i v}\right\}$. 


\section{B. Discretization Method}

In order to illustrate a strategy to define an array with the same NDF of the corresponding continuous current source, we, now, consider the far zone case and show the proposed approach with reference to the source domain. It is based on the consideration of the PSF function since, by its very definition, it is able to reconstruct reliably two close point-like sources. This can be achieved successfully when the two sources are adequately spaced away each other. In particular, the PSF main lobe width may provide a quantitative measure of the adequate spacing. Accordingly, an intuitive procedure to establish the locations of the sources can be established as the maxima of the main lobes of the normalized PSFs, when they overlap at a prefixed level $\kappa_{i}$ (see Fig. 10). Accordingly, the centers of the main bells of the PSFs provide the points where to discretize the source to ensure a resolution equal to the width of the normalized PSF main lobe at the height $\kappa_{i}$. In order to perform this procedure by analytical expressions, we adopt the approximate evaluations of the PSFs of previous Section by (16), whose accuracy has been demonstrated within the relevant main lobe.

We point out explicitly that, as the PSFs are spatially variant, this procedure will result in a non-uniform discretization. In addition, the chosen value of $\kappa_{i}$ plays an important role in defining the number of discrete sources: the higher the value of $\kappa_{i}$, the higher the level where the PSFs' main lobes will overlap and, accordingly, the higher the number of discrete points obtained (see Fig. 10).
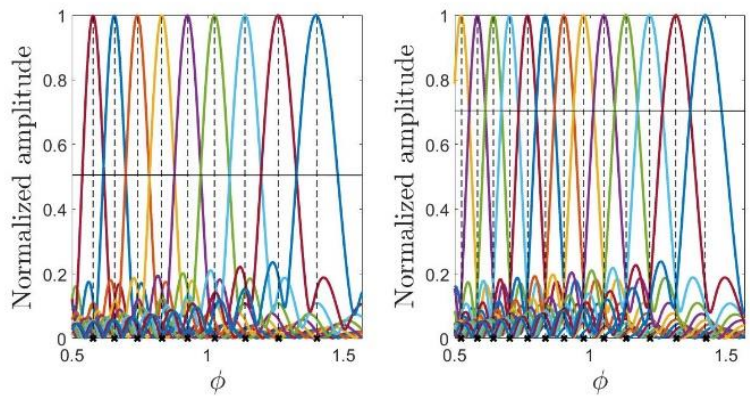

Fig. $10 \mathrm{~A}$ pictorial example of the behavior of the normalized $\widetilde{P S F_{F}} S$ overlapped along part of the source domain. The black crosses on the horizontal axis identify the resulting centers of the main lobes and, therefore, the points of discretization. The higher the level $\kappa_{F}$ (right panel) the higher the number of points.

The adopted strategy, then, can be depicted as follows:

- we choose the $\epsilon_{i}$ truncation level of the normalized SVs of the $\mathcal{A}_{i}$ operator in (3) so to fix the relevant NDF of the problem;

- we fix $\kappa_{i}$, where the PSFs main lobes overlap, at a rather high value;

- we identify the discretization points as the centers of the main lobes of the approximate PSFs overlapping at the $\kappa_{i}$ level along the full source domain;

- we compute the SVs of the operator, say $\tilde{\mathcal{A}}_{i}$, mapping the resulting discrete source into the corresponding far field;

- if the behavior of the corresponding normalized singular values is not flat, the $\kappa_{i}$ level is further lowered since this means that the found number of point-like sources is higher than the NDF;

- we return to the previous steps and identify a new set of discretization points and the last steps are repeated until the achieved discretization returns for $\tilde{\mathcal{A}}_{i}$ a singular values flat behavior.

This procedure ensures that the number of point-like sources is equal to the NDF of the corresponding continuous source, so that both the dimension of the set of radiated fields are equal. Of course, the actual sets may differ, but the requirement of a common dimension can be adopted to establish a meaningful comparison between their radiated fields.

The relevance of the non-uniform spacing in discretization of the operators (3) can be appreciated by the numerical results shown in Fig. 11. In fact, the behavior of the singular values of operators (20) are displayed in comparison with the ones of the continuous source case, with the goal of defining an array geometry whose radiated field belongs to a functional subspace with the same dimension (i.e. the number of significant singular values before the step) of the continuous case. The source geometry consists in an arc of circumference with $\mathrm{R}=10 \lambda$ and $\alpha=\pi / 2$ observed for $\gamma=3 \pi / 8$ in far zone and for $\mathrm{R}_{\mathrm{o}}=15 \lambda$ and $\gamma=3 \pi / 8$ in near zone, respectively. For the far zone case, indeed, the number of significant singular values of both $\mathcal{A}_{F}$ and $\tilde{\mathcal{A}}_{F}$ in the NU case is 43 , whereas for $\tilde{\mathcal{A}}_{F}$ in the $\mathrm{U}$ case the number decreases to 37. For the near zone, the NDF of both $\mathcal{A}_{N}$ and $\tilde{\mathcal{A}}_{N}$ (NU case) is 47 and reduces to 40 when the points are uniformly spaced.

On the other hand, if we would obtain the same source subspace dimension by a discrete source with uniform spacing, we should increase the number of radiating elements so implying a more complex source and additional costs. In fact, for the examples of Fig. 11, $N A=57$ is required so that $\tilde{\mathcal{A}}_{F}$ admits 43 significant singular values and $N A=65$ is required so that $\tilde{\mathcal{A}}_{N}$ admits 47 significant singular. In any case the number of elements required would increase with respect to the non-uniform step, by $32 \%$ and $38 \%$, respectively.

This means that a non-uniform source discretization is more efficient than a uniform one, since it requires a lower number of array elements for a circumference arc geometry, when the radiated field is observed along a limited angular domain.
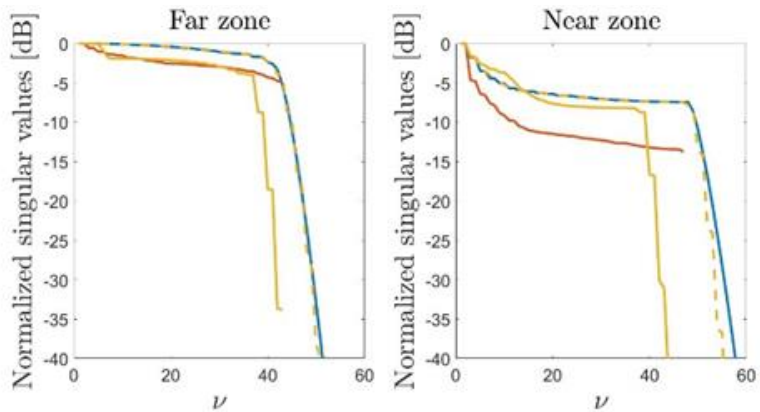

Fig. 11 Comparison of the normalized singular values (left panel) of $\mathcal{A}_{F}$ for the continuous source (blue line), of $\tilde{\mathcal{A}}_{F}$ for the non-uniform case (red line), of $\tilde{\mathcal{A}}_{F}$ for the uniform spacing with the same number of array elements (yellow line). The right panel displays the same results for near zone case for the same geometries as in Fig. 4. The dashed yellow curve refers to a uniform array with the minimum number of elements (57 and 67 for the far and the near zone, respectively) needed to achieve the NDF of the continuous case. Since $\epsilon_{F}=$ 
$-5 d B$ is chosen for the far zone and $\epsilon_{N}=-14 d B$ for the near zone, it results $\kappa_{F}=0.65$ and $\kappa_{N}=0.5$, respectively.
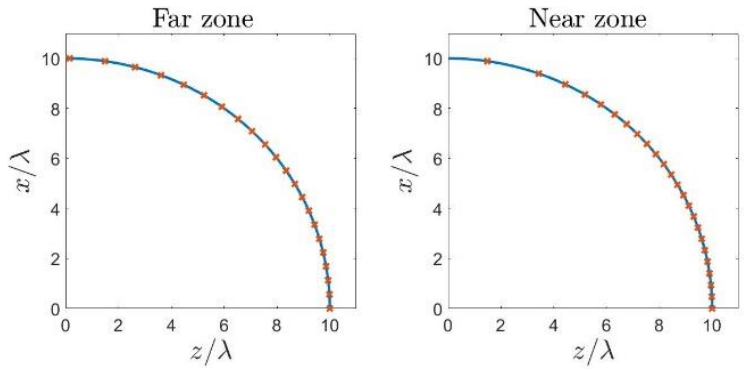

Fig. 12 The non-uniform array configurations corresponding to the SVs of the geometries of Fig. 11 in the far (left panel) and near (right panel) cases. The discrete source positions are depicted red crosses. For symmetry reasons, only the upper half of the array sources are shown.

It can be pointed out that the discretization procedure leads to a non-uniform spacing between the elements of a discrete (array) source (Fig. 12) so that it is worth to investigate how this affects the array performance especially in comparison with a standard uniform array with the same number of source points.

\section{Numerical APPlications to ArRay Problems}

The previous Sections have been devoted to apply an inverse problem approach to the analysis of the radiation operator by means of its SVD, when the field observation domain is limited. The singular values behavior defines the dimension of the relevant sets of fields that can be radiated, through the evaluation of the NDF of the source. The PSF analysis leads to a procedure to define the optimal locations of a discrete source whose set of radiated field has the same dimension as the one of the corresponding continuous circumference arc geometry. These results can be very useful for conformal arrays since general analytical methods lack for their design.

Therefore, in this section, we show how the NU array allows to achieve better performances and results closer to those of the corresponding continuous source than the $\mathrm{U}$ array. The first two applications are concerned with the synthesis of a focusing beam and the diagnosis of the faulty elements within the array. To this end we refer to same source geometries considered in Fig. 11 of the previous Section. So, the source (both in the continuous and the discrete cases) is supposed to be supported over a semi-circumference of radius $R=10 \lambda$. Because of the results shown in Fig. 7, which allows to define the minimum number of array elements in the NU case, we choose to compare this NU array with a uniform one with the same number of elements, but evenly spaced.

\section{A. Array Synthesis}

First, we deal with a pattern synthesis problem so to consider the far zone operators. According to the result of Fig. 11, a NU array composed of $N A=43$ elements radiating a pattern complying with (4) (see left panel of Fig. 12) is able to provide a set of far fields with the same dimension as the continuous source of the same geometry. Therefore, the $\mathrm{U}$ array to be considered for the purpose of comparison is composed of the same number of equispaced elements at $\Delta \phi=0.0748 \mathrm{rad}$, corresponding to $0.748 \lambda$. Since in some applications, as in surveillance radar, it is required to radiate identical beams pointing at different directions, a conformal source can achieve a uniform coverage over an angular range larger than a line array, we require a sinc-like focusing beam with main lobe amplitude $\Delta \theta=0.142 \mathrm{rad}$

$U_{D}(\theta)=\operatorname{sinc}\left[\frac{2 \pi}{\Delta \theta}\left(\theta-\theta_{0}\right)\right]$,

where $\theta_{0}$ is the direction where the beam maximum is to be pointed.

The synthesis procedure amounts to projecting the assigned far field onto the relevant left singular functions

$\tilde{E}_{F}(\theta)=\sum_{v=1}^{N(\epsilon)}\left\langle U_{D}(\theta), \tilde{u}_{F v}(\theta)\right\rangle \tilde{u}_{F v}(\theta)$.

The amplitude of these projections is plotted in Fig. 13 and shows that the NU array configuration provides the better results in terms of beams uniformity. For the sake of comparison, also the results of a continuous source of the same length as the discrete one are reported, since they can be assumed as a reference for the array radiation.

In addition, the directivity of the projected beams is computed as

$D\left(\theta_{0}\right)=\frac{\left|\tilde{E}_{F}\left(\theta_{0}\right)\right|^{2}}{\frac{1}{2 \gamma} \int_{-\gamma}^{\gamma}\left|\tilde{E}_{F}(\theta)\right|^{2} d \theta}$

under Table I. By comparing it with the continuous source case as well, again the better results are obtained for the $N U$ configuration, since the directivity values are more similar also for the off-set maximum direction.
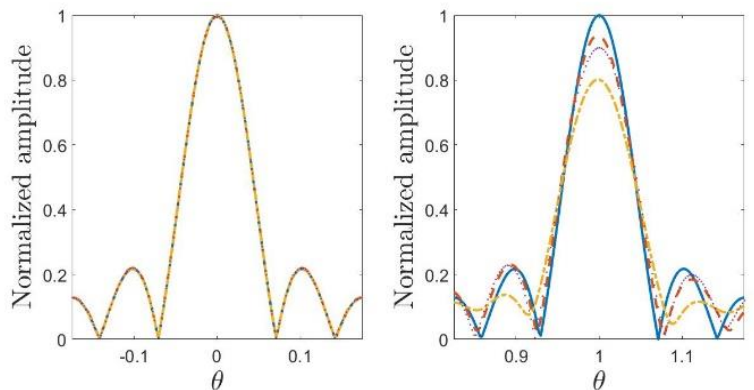

Fig. 13 The amplitude of $U_{D}(\theta)$ (blue line), of $\widetilde{E}_{F}(\theta)$ for the NU (red line) and $\mathrm{U}$ (yellow line) array configurations and of $\operatorname{EF}(\theta)$ (violet dotted line) for the continuous source.

TABLE I

$D\left(\theta_{0}\right)$ VARYING THE BEAM MAXIMUM ANGLE $\theta_{0}$

\begin{tabular}{ccc}
\hline \hline$\theta_{0}$ & $0 \mathrm{rad}$ & $1 \mathrm{rad}$ \\
\hline Continuous source & $30.4 \mathrm{~dB}$ & $29.3 \mathrm{~dB}$ \\
$N U$ array & $30.4 \mathrm{~dB}$ & $29.8 \mathrm{~dB}$ \\
$U$ array & $30.5 \mathrm{~dB}$ & $28.4 \mathrm{~dB}$ \\
\hline \hline
\end{tabular}

\section{B. Array Diagnostic}

In order to consider the application of the results of the previous Sections to the near field zone, we consider an array diagnostic problem from near zone data, collected over a 
limited angular domain and compare the two array configurations again, i.e. the one with non-uniform element spacing and the one with uniform spacing. We assume that some array elements may develop an "on-off" fault, so the faulty elements do not radiate at all. This type of fault is common in practical applications when mechanical defects may prevent the feeding of antenna elements. We assume, then, that the $\mathrm{NU}$ array configuration correspond to the result shown in the right panel of Fig. 12, namely it is composed of $N A=47$ elements, while the $\mathrm{U}$ configuration consists of the same number of elements spaced at $\Delta \phi=0.0683 \mathrm{rad}$. Both arrays are designed to radiate a focusing field at the direction $\theta_{0}=0$, that is, their elements are fed by currents with constant modulus and different phases

$I_{l}=e^{-j \beta R \cos \phi_{l}^{k}}$.

The radiated field measurements are collected in near zone and corrupted by a complex gaussian noise with zero mean such to assure a signal-to-noise ratio (SNR) of $20 \mathrm{~dB}$. Once the radiated field data are gathered, the source is reconstructed by a TSVD algorithm as

$\underline{\hat{I}}=\sum_{v=1}^{N\left(\epsilon_{N}\right) \leq E, \tilde{u}_{N v}^{k}>} \widetilde{\tilde{v}}_{N v}^{k}$,

with $k \in\{N U, U\}$.

From the results of Fig. 14, one can clearly see that the faulty elements are well detected only in the $N U$ configuration. This result is also quantitatively confirmed by the values shown in Table II reporting the relative error defined as

$$
e^{k}=\frac{\|\underline{I}-\underline{I}\|}{\|\underline{I}\|}
$$

where $\underline{I}^{k}$ stands for the actual excitation vector.

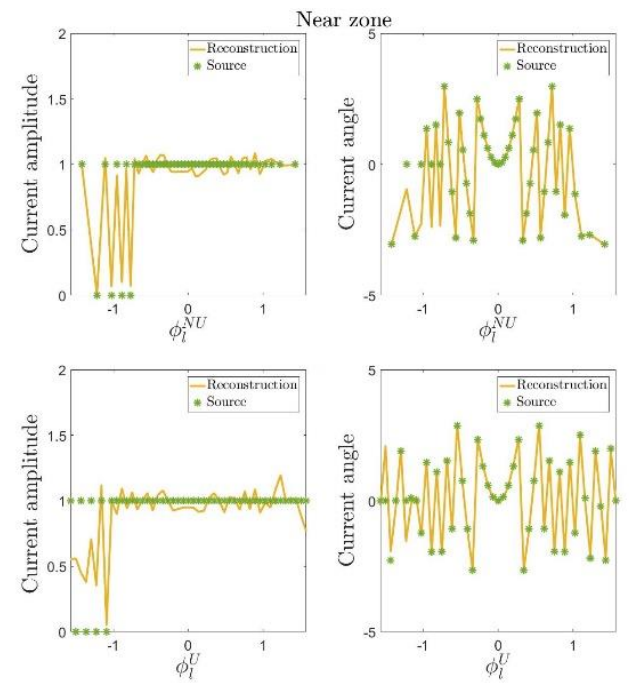

Fig. 14 Source reconstruction for a $N U$ (upper panel) and a $U$ (lower panel) configuration with noisy data $\left(S N R_{d B}=20 d B\right)$ collected in near zone. The source and observation geometries are the same as in Figs. 11 and 12.
TABLE II

NORMALIZED RECONSTRUCTION ERROR FOR NEAR ZONE OBSERVATIONS.

\begin{tabular}{cc}
\hline \hline$e^{N U}$ & $e^{U}$ \\
\hline $7.6710^{-2}$ & $1.9910^{-1}$ \\
\hline
\end{tabular}

\section{CONCLUSION}

The role of a limited observation domain in the radiation of conformal sources has been highlighted by referring to a $2 \mathrm{D}$ circumference geometry. The analysis has been carried out by the investigation the SVD of the relevant operator and the resulting PSFs. The influence of an appropriate weight function, connected to the source element factor, has been examined in achieving an approximate closed form expression of the PSF.

Then, the main consequence of the limited observation domain on the PSF behavior has been emphasized, i.e. the dependence of the PSF on its maximum direction. This has led to its angularly variant behavior whose relevance in not only mathematical, but also has several practical applications in antenna analysis and synthesis.

To this end, a source discretization strategy has been introduced, leading to an optimal non-uniform spacing of an array radiating a field with the same NDF of the continuous source. The comparison of its performance with the case of an equispaced array of the same number of elements has revealed better performances both in the synthesis of radiation pattern and in the diagnostics of faulty elements.

\section{APPENDIX A}

In the general case of source and field functions belonging to the set of square integrable functions $L^{2}$ supported over different intervals as in (1), the singular functions are solution of the following shifted eigenvalue problem introduced by Lanczos [17]

$\mathcal{A}_{i} v_{i v}=\sigma_{i v} u_{i v}$

$\mathcal{A}_{i}^{\dagger} u_{i v}=\sigma_{i v} v_{i v}$,

where $i=F$ or $N$.

When source and field functions are supported on the same full angle circumference observed on a full angle, the eigenfunctions are of exponential type and we can derive the analytic expressions of the eigenvalues for both the far and the near zone for different weight functions (4).

Let us consider the equation (A1) for the far zone and show that, by choosing $v_{F n}(\phi)=e^{j n \phi}$ and $u_{F n}(\theta)=e^{j n \theta}$, (A1) is satisfied. In fact, by direct substitution

$$
\begin{gathered}
\mathcal{A}_{F} e^{j n \phi}=R \int_{-\pi}^{\pi} e^{j n \phi} e^{j \beta R \cos (\theta-\phi)} \\
\cdot(1+\cos (\theta-\phi))^{m} d \phi
\end{gathered}
$$

and application of the Jacobi-Anger expansion of the exponential function and of the half-angle formula for the 
cosine, we obtain

$$
\begin{gathered}
2^{m} R \sum_{p=-\infty}^{+\infty} j^{p} J_{p}(\beta R) e^{j p \theta} . \\
\cdot \int_{-\pi}^{\pi} e^{j(n-p) \phi} \cos ^{2 m}\left(\frac{\theta-\phi}{2}\right) d \phi
\end{gathered}
$$

Next, the Euler's formula and the application of the binomial theorem

$(a+b)^{m}=\sum_{k=0}^{m}\left(\begin{array}{c}m \\ k\end{array}\right) a^{m-k} b^{k}$,

leads to

$2^{-m} R \sum_{p=-\infty}^{+\infty} j^{p} J_{p}(\beta R) e^{j p \theta} \sum_{k=0}^{2 m}\left(\begin{array}{c}2 m \\ k\end{array}\right)$.

$\cdot \int_{-\pi}^{\pi} e^{j(n-p) \phi} e^{j(2 m-k)\left(\frac{\theta-\phi}{2}\right)} e^{-j k\left(\frac{\theta-\phi}{2}\right)} d \phi$

If we single out of the integral the terms that do not depend on $\phi$, the resulting integral is elementary and is different from zero only if $p=n+k-m$. Accordingly, (A6) becomes

$\mathcal{A}_{F} e^{j n \phi}=$
$2^{-m+1} \pi R \sum_{k=0}^{2 m}\left(\begin{array}{c}2 m \\ k\end{array}\right) j^{n+k-m} J_{n+k-m}(\beta R) e^{j n \theta} \cdot$

Therefore, the eigenvalues (5) are recovered.

For $m=0$, it is straightforward to obtain the known result

$\lambda_{F n}=2 \pi R j^{n} J_{n}(\beta R)$.

For larger values of $m$ the expression (5) can become complicated, but thanks to the recurrence relations of the Bessel functions we can simplify it and, consequently, the $n-$ th eigenvalue turns out a combination of the $n$-th Bessel functions and its derivatives in a more compact form. Let us show this for the first values of $m$.

For $m=1,(5)$ returns

$\lambda_{F n}=2 \pi R j^{n}\left[-\frac{j}{2} J_{n-1}(\beta R)+J_{n}(\beta R)+\frac{j}{2} J_{n+1}(\beta R)\right]$.

Eq. (A9) can be recast as

$\lambda_{F n}=2 \pi R j^{n}\left[J_{n}(\beta R)-j J^{\prime}{ }_{n}(\beta R)\right]$,

where $J_{n}^{\prime}(\cdot)$ is the derivative of the Bessel function of $n-$ th order with respect to its argument.

For $m=2,(5)$ returns

$$
\begin{aligned}
\lambda_{F n}=6 \pi R j^{n} & {\left[-\frac{1}{12} J_{n-2}(\beta R)-\frac{j}{3} J_{n-1}(\beta R)+\frac{1}{2} J_{n}(\beta R),\right.} \\
+ & \left.\frac{j}{3} J_{n+1}(\beta R)-\frac{1}{12} J_{n+2}(\beta R)\right]
\end{aligned}
$$

and, again, by resorting to the recurrence relations of the Bessel functions we finally obtain

$$
\lambda_{F n}=\begin{gathered}
2 \pi R j^{n}\left[\left(2-\left(\frac{n}{\beta R}\right)^{2}\right) J_{n}(\beta R)+\right. \\
\left.+\left(\frac{1}{\beta R}-j 2\right) J_{n}^{\prime}(\beta R)\right]
\end{gathered} .
$$

Let us, now, repeat the same reasoning for the near zone. If the near zone radiation operator is applied to the exponential function $e^{j n \phi}$, we get

$$
\begin{aligned}
& \mathcal{A}_{N} e^{j n \phi}= R \int_{-\pi}^{\pi} e^{j n \phi} H_{0}^{(2)}(\beta|\underline{r}(\theta)-\underline{r}(\phi)|) . \\
& \cdot(1+\cos (\theta-\phi))^{m} d \phi
\end{aligned} .
$$

By exploiting the addition theorem for the Hankel functions related to the case $R_{o}>R$, namely,

$$
\begin{gathered}
H_{0}^{(2)}(\beta|\underline{r}(\theta)-\underline{r}(\phi)|)= \\
=\sum_{p=-\infty}^{+\infty} J_{p}(\beta R) H_{p}^{(2)}\left(\beta R_{o}\right) e^{j p(\theta-\phi)},
\end{gathered}
$$

and the same mathematical details as before, except for the presence of the Hankel function, we reach (6).

Let us now particularize the expression of the eigenvalues for the case $m=0, m=1$ and $m=2$.

For $m=0$ (6) easily returns the known result

$\lambda_{N n}=2 \pi R J_{n}(\beta R) H_{n}^{(2)}\left(\beta R_{o}\right)$.

If $m=1$, instead, the eigenvalues have a little more complicated expression since they write as

$$
\begin{gathered}
\lambda_{N n}=2 \pi R\left[\frac{1}{2} J_{n-1}(\beta R) H_{n-1}^{(2)}\left(\beta R_{o}\right)+\right. \\
\left.J_{n}(\beta R) H_{n}^{(2)}\left(\beta R_{o}\right)+\frac{1}{2} J_{n+1}(\beta R) H_{n+1}^{(2)}\left(\beta R_{o}\right)\right]
\end{gathered} .
$$

In order to provide a more compact form, like before, we make use of the recurrence formulas of the Bessel/Hankel function as a function of the corresponding Bessel/Hankel function of $n$-th order and its derivative.

This allow us to recast (A16) as

$$
\begin{gathered}
\lambda_{N n}=2 \pi R\left[\frac{1}{2}\left(J_{n}^{\prime}(\beta R)+\frac{n}{\beta R} J_{n}(\beta R)\right) .\right. \\
\cdot\left(H_{n}^{(2) \prime}\left(\beta R_{o}\right)+\frac{n}{\beta R_{o}} H_{n}^{(2)}\left(\beta R_{o}\right)\right)+ \\
J_{n}(\beta R) H_{n}^{(2)}\left(\beta R_{o}\right)+\frac{1}{2}\left(-J_{n}^{\prime}(\beta R)+\frac{n}{\beta R} J_{n}(\beta R)\right) . \\
\left.\cdot\left(-H_{n}^{(2) \prime}\left(\beta R_{o}\right)+\frac{n}{\beta R_{o}} H_{n}^{(2)}\left(\beta R_{o}\right)\right)\right]
\end{gathered}
$$

Combining together the similar terms, we finally get

$$
\begin{gathered}
\lambda_{N n}=2 \pi R\left[J_{n}^{\prime}(\beta R) H_{n}^{(2) \prime}\left(\beta R_{o}\right)+\right. \\
\left.\left(\frac{n^{2}}{\beta^{2} R R_{o}}+1\right) J_{n}(\beta R) H_{n}^{(2)}\left(\beta R_{o}\right)\right]
\end{gathered}
$$


When $m=2$, we obtain the following expression for the eigenvalues

$$
\begin{gathered}
\lambda_{N n}=\pi R\left\{4\left(\frac{n^{2}+1}{\beta^{2} R R_{o}}+1\right) J_{n}^{\prime}(\beta R) H_{n}^{(2) \prime}\left(\beta R_{o}\right)+\right. \\
+2\left(\frac{1}{\beta R}-\frac{4 n^{2}}{\beta^{3} R R_{o}^{2}}\right) J_{n}^{\prime}(\beta R) H_{n}^{(2)}\left(\beta R_{o}\right)+ \\
+2\left(\frac{1}{\beta R_{o}}-\frac{4 n^{2}}{\beta^{3} R^{2} R_{o}}\right) J_{n}(\beta R) H_{n}^{(2) \prime}\left(\beta R_{o}\right)+ \\
\left.\left(4+\frac{2 n^{2}\left[2 n^{2}+2+\beta^{2}\left(R_{o}^{2}-R^{2}+2 R R_{o}\right)\right]}{\left(\beta^{2} R R_{o}\right)^{2}}\right) J_{n}(\beta R) H_{n}^{(2)}\left(\beta R_{o}\right)\right\}
\end{gathered}
$$

\section{APPENDIX B}

Hereafter, we derive the analytical expression of the approximate PSF for operators $\mathcal{A}_{F}$ e $\mathcal{A}_{N}$. For the far zone, (14) writes

$$
\begin{gathered}
{\widetilde{P S F_{F}}}\left(\phi, \phi_{0}\right)=R^{2} \int_{-\gamma}^{\gamma} e^{-j \beta R \cos (\theta-\phi)} . \\
\cdot(1+\cos (\theta-\phi))^{m} \int_{-\alpha}^{\alpha} e^{j \beta R \cos \left(\theta-\phi^{\prime}\right)} \cdot \\
\cdot\left(1+\cos \left(\theta-\phi^{\prime}\right)\right)^{m} \delta\left(\phi^{\prime}-\phi_{0}\right) d \phi^{\prime} d \theta
\end{gathered}
$$

By applying the sifting property of delta function, (B1) becomes

$$
\begin{gathered}
\widetilde{P S F_{F}}\left(\phi, \phi_{0}\right)=R^{2} \int_{-\gamma}^{\gamma} e^{j \beta R\left[\cos \left(\theta-\phi_{0}\right)-\cos (\theta-\phi)\right]} . \\
\cdot(1+\cos (\theta-\phi))^{m}\left(1+\cos \left(\theta-\phi_{0}\right)\right)^{m} d \theta
\end{gathered},
$$

and, by using the sum-to-product formula to the exponential term, we get

$$
\begin{gathered}
{\widetilde{P S F_{F}}}_{F}\left(\phi, \phi_{0}\right)=R^{2} \int_{-\gamma}^{\gamma} e^{j 2 \beta R \sin \left(\frac{\phi_{0}-\phi}{2}\right) \sin \left(\theta-\frac{\phi+\phi_{0}}{2}\right)} . \\
\cdot(1+\cos (\theta-\phi))^{m}\left(1+\cos \left(\theta-\phi_{0}\right)\right)^{m} d \theta
\end{gathered} .
$$

At this point, it is possible to use the Jacobi-Anger expansion for the exponential in order to obtain

$$
\begin{gathered}
\widetilde{P S F_{F}}\left(\phi, \phi_{0}\right)=R^{2} \int_{-\gamma}^{\gamma} \sum_{n=-\infty}^{+\infty} J_{n}\left(2 \beta R \sin \left(\frac{\phi_{0}-\phi}{2}\right)\right) . \\
\cdot e^{j n\left(\theta-\frac{\phi+\phi_{0}}{2}\right)}(1+\cos (\theta-\phi))^{m} . \\
\cdot\left(1+\cos \left(\theta-\phi_{0}\right)\right)^{m} d \theta
\end{gathered},
$$

where $J_{n}(\cdot)$ is the Bessel function of the first kind and $n-$ th order. Then, applying the half-angle and Euler's formula to the cosine terms, we can state that

$$
\begin{gathered}
\widetilde{\operatorname{PSF}_{F}}\left(\phi, \phi_{0}\right)=R^{2} 2^{-2 m} . \\
\cdot \int_{-\gamma}^{\gamma} \sum_{n=-\infty}^{+\infty} J_{n}\left(2 \beta R \sin \left(\frac{\phi_{0}-\phi}{2}\right)\right) e^{j n\left(\theta-\frac{\phi+\phi_{0}}{2}\right)} . \\
\cdot\left(e^{j \frac{\theta-\phi}{2}}+e^{-j \frac{\theta-\phi}{2}}\right)^{2 m}\left(e^{j \frac{\theta-\phi_{0}}{2}}+e^{-j \frac{\theta-\phi_{0}}{2}}\right)^{2 m} d \theta
\end{gathered}
$$

$$
\begin{gathered}
\widetilde{\operatorname{PSF}_{F}}\left(\phi, \phi_{0}\right)=R^{2} 2^{-2 m} e^{-j m\left(\phi+\phi_{0}\right)} . \\
\cdot \sum_{n=-\infty}^{+\infty} J_{n}\left(2 \beta R \sin \left(\frac{\phi_{0}-\phi}{2}\right)\right) e^{-j n \frac{\phi+\phi_{0}}{2}} . \\
\cdot \sum_{k=0}^{2 m}\left(\begin{array}{c}
2 m \\
k
\end{array}\right) e^{j k \phi} \sum_{s=0}^{2 m}\left(\begin{array}{c}
2 m \\
s
\end{array}\right) e^{j s \phi_{0}} \int_{-\gamma}^{\gamma} e^{j(n+2 m-k-s)} d \theta
\end{gathered}
$$

The integral, now, is easily solvable and leads to the following final expression for the approximate PSF

$$
\begin{gathered}
\widetilde{P S F_{F}}\left(\phi, \phi_{0}\right)=\gamma R^{2} 2^{-2 m+1} e^{-j m\left(\phi+\phi_{0}\right)} . \\
\cdot \sum_{n=-\infty}^{+\infty} J_{n}\left(2 \beta R \sin \left(\frac{\phi_{0}-\phi}{2}\right)\right) e^{-j n \frac{\phi+\phi_{0}}{2}} \sum_{k=0}^{2 m}\left(\begin{array}{c}
2 m \\
k
\end{array}\right) . \\
\cdot e^{j k \phi} \sum_{s=0}^{2 m}\left(\begin{array}{c}
2 m \\
s
\end{array}\right) e^{j s \phi_{0}} \operatorname{sinc}[(n+2 m-k-s) \gamma]
\end{gathered}
$$

We can point out that for $m=0$ the expression just found reduces to

$$
\begin{aligned}
\widetilde{P S F_{F}}\left(\phi, \phi_{0}\right)= & 2 \gamma R^{2} \sum_{n=-\infty}^{+\infty} J_{n}\left(2 \beta R \sin \left(\frac{\phi_{0}-\phi}{2}\right)\right) . \\
& \cdot e^{-j n \frac{\phi+\phi_{0}}{2}} \operatorname{sinc}[n \gamma]
\end{aligned}
$$

When $\gamma=\pi$, the sinc function in (B7) has a non-null contribution only for $n=k+s-2 m$ and the resulting approximate PSF depends on the difference $\phi-\phi_{0}$

$$
\begin{aligned}
& \widetilde{P S F_{F}}\left(\phi, \phi_{0}\right)=\pi R^{2} 2^{-2 m+1} \sum_{k=0}^{2 m}\left(\begin{array}{c}
2 m \\
k
\end{array}\right) e^{j \frac{k}{2}\left(\phi-\phi_{0}\right)} . \\
& \cdot \sum_{s=0}^{2 m}\left(\begin{array}{c}
2 m \\
s
\end{array}\right) e^{-j \frac{s}{2}\left(\phi-\phi_{0}\right)} J_{k+s-2 m}\left(2 \beta R \sin \left(\frac{\phi_{0}-\phi}{2}\right)\right)
\end{aligned}
$$

that is, it is spatially invariant. We expect this property to hold even for $\gamma$ approaching $\pi$, since the sinc terms adding in (B7) become smaller and smaller when the indices' sum in the argument grows in module, so that, again, the main contribution is for $n=k+s-2 m$.

As far as the near zone is concerned, (14) is made explicit as

$$
\begin{gathered}
\widetilde{P S F_{N}}\left(\phi, \phi_{0}\right)=R^{2} \int_{-\gamma}^{\gamma} H_{0}^{(2)^{*}}(\beta|\underline{r}(\theta)-\underline{r}(\phi)|) \cdot \\
\cdot(1+\cos (\theta-\phi))^{m} \int_{-\alpha}^{\alpha} H_{0}^{(2)}\left(\beta\left|\underline{r}(\theta)-\underline{r}\left(\phi^{\prime}\right)\right|\right) \cdot \\
\cdot\left(1+\cos \left(\theta-\phi^{\prime}\right)\right)^{m} \delta\left(\phi^{\prime}-\phi_{0}\right) d \phi^{\prime} d \theta
\end{gathered}
$$

that, thanks to the sifting property of the delta function, becomes

$$
\begin{gathered}
\widetilde{P S F_{N}}\left(\phi, \phi_{0}\right)=R^{2} \int_{-\gamma}^{\gamma} H_{0}^{(2)^{*}}(\beta|\underline{r}(\theta)-\underline{r}(\phi)|) \cdot \\
\cdot(1+\cos (\theta-\phi))^{m} H_{0}^{(2)}\left(\beta\left|\underline{r}(\theta)-\underline{r}\left(\phi_{0}\right)\right|\right) \cdot \cdot \\
\cdot\left(1+\cos \left(\theta-\phi_{0}\right)\right)^{m} d \theta
\end{gathered}
$$

By exploiting the half-angle formula and the Euler's formula in order to cast the cosines in form of exponentials, the previous expression turns into 


$$
\begin{gathered}
\widetilde{\operatorname{PSF}_{N}}\left(\phi, \phi_{0}\right)=R^{2} 2^{-2 m} \int_{-\gamma}^{\gamma} H_{0}^{(2)^{*}}(\beta|\underline{r}(\theta)-\underline{r}(\phi)|) . \\
\cdot\left(e^{j \frac{\theta-\phi}{2}}+e^{-j \frac{\theta-\phi}{2}}\right)^{2 m} H_{0}^{(2)}\left(\beta\left|\underline{r}(\theta)-\underline{r}\left(\phi_{0}\right)\right|\right) \cdot . \\
\cdot\left(e^{j \frac{\theta-\phi_{0}}{2}}+e^{-j \frac{\theta-\phi_{0}}{2}}\right)^{2 m} d \theta
\end{gathered}
$$

Once again, resorting to the binomial theorem and singling out of the integral the term which does not depend on $\theta$, we come to

$$
\begin{gathered}
\widetilde{P S F_{N}}\left(\phi, \phi_{0}\right)=R^{2} 2^{-2 m} e^{-j m\left(\phi+\phi_{0}\right)} . \\
\cdot \int_{-\gamma}^{\gamma} H_{0}^{(2)^{*}}(\beta|\underline{r}(\theta)-\underline{r}(\phi)|) H_{0}^{(2)}\left(\beta\left|\underline{r}(\theta)-\underline{r}\left(\phi_{0}\right)\right|\right) \cdot \\
\cdot e^{j 2 m \theta} \sum_{k=0}^{2 m}\left(\begin{array}{c}
2 m \\
k
\end{array}\right) e^{-j k(\theta-\phi)} \sum_{s=0}^{2 m}\left(\begin{array}{c}
2 m \\
s
\end{array}\right) e^{-j s\left(\theta-\phi_{0}\right)} d \theta
\end{gathered}
$$

and then, by exploiting the addition theorem (A14) of Hankel functions for $R_{o} \geq R$ and substituting it into (B13), the PSF becomes

$$
\begin{gathered}
\widetilde{P S F_{N}}\left(\phi, \phi_{0}\right)=R^{2} 2^{-2 m} e^{-j m\left(\phi+\phi_{0}\right)} \\
\cdot \sum_{p=-\infty}^{+\infty} J_{p}(\beta R) H_{p}^{(2) *}\left(\beta R_{o}\right) e^{j p \phi} \\
\cdot \sum_{l=-\infty}^{+\infty} J_{l}(\beta R) H_{l}^{(2)}\left(\beta R_{o}\right) e^{-j l \phi_{0}} \sum_{k=0}^{2 m}\left(\begin{array}{c}
2 m \\
k
\end{array}\right) e^{j k \phi} \cdot \\
\cdot \sum_{s=0}^{2 m}\left(\begin{array}{c}
2 m \\
S
\end{array}\right) e^{j s \phi_{0}} \int_{-\gamma}^{\gamma} e^{j(2 m-p+l-k-s) \theta} d \theta
\end{gathered}
$$

The latter integral, at this point, is easily resolved and we get the final expression of the approximate PSF

$$
\begin{gathered}
\widehat{P S F_{N}}\left(\phi, \phi_{0}\right)=\gamma R^{2} 2^{-2 m+1} e^{-j m\left(\phi+\phi_{0}\right)} \sum_{p=-\infty}^{+\infty} J_{p}(\beta R) \cdot \\
\cdot H_{p}^{(2) *}\left(\beta R_{o}\right) e^{j p \phi} \text { onlye }{ }^{-j l \phi_{0}} \sum_{k=0}^{2 m}\left(\begin{array}{c}
2 m \\
k
\end{array}\right) e^{j k \phi} . \\
\cdot \sum_{s=0}^{2 m}\left(\begin{array}{c}
2 m \\
s
\end{array}\right) e^{j s \phi_{0}} \operatorname{sinc}[(2 m-p+l-k-s) \gamma]
\end{gathered}
$$

Finally, we notice that in absence of weight $(m=0)$ the expression (B15) is reduced to

$$
\begin{gathered}
\widetilde{P S F_{N}}\left(\phi, \phi_{0}\right)=2 \gamma R^{2} \sum_{p=-\infty}^{+\infty} J_{p}(\beta R) H_{p}^{(2) *}\left(\beta R_{o}\right) . \\
\cdot \sum_{l=-\infty}^{+\infty} J_{l}(\beta R) H_{l}^{(2)}\left(\beta R_{o}\right) e^{j\left(p \phi-l \phi_{0}\right)} \operatorname{sinc}[(l-p) \gamma]
\end{gathered}
$$

We point out that, again, spatial invariance is found when $\gamma=\pi$ in (B15) since the main contribution in the sinc summation would occur for $l=p+k+s-2 m$, leading for $\gamma=\pi$ to the following expression of the approximate PSF

$$
\begin{gathered}
\widetilde{P S F_{N}}\left(\phi, \phi_{0}\right)=\pi R^{2} 2^{-2 m+1} e^{-j m\left(\phi-\phi_{0}\right)} \\
\cdot \sum_{p=-\infty}^{+\infty} J_{p}(\beta R) H_{p}^{(2) *}\left(\beta R_{o}\right) e^{j p\left(\phi-\phi_{0}\right)} \\
\cdot \sum_{k=0}^{2 m}\left(\begin{array}{c}
2 m \\
k
\end{array}\right) e^{j k\left(\phi-\phi_{0}\right)} \sum_{s=0}^{2 m}\left(\begin{array}{c}
2 m \\
s
\end{array}\right) e^{j s\left(\phi-\phi_{0}\right)} \cdot \\
\cdot J_{p+k+s-2 m}(\beta R) H_{p+k+s-2 m}^{(2)}\left(\beta R_{o}\right)
\end{gathered}
$$

which depends only on the difference between the point source angle $\phi_{0}$ and the observation one $\phi$. The same holds true approximately also for $\gamma$ approaching $\pi$ since the main contribution to s summation comes from the same $l=p+k+$ $s-2 m$ index.

\section{REFERENCES}

[1] H. Orazi, H. Soleimani, and R. Azadkhah, "Optimum pattern synthesis of non-uniform spherical arrays using the Euler rotation," IET Microw. Antennas Propag., vol. 9, no. 9, pp. 898-904, 2015.

[2] W. Li, Y. Hei, J. Yang, and X. Shi, "Optimisation of nonuniform time-modulated conformal arrays using an improved non-dominated sorting genetic-II algorithm," IET Microw. Antennas Propag., vol. 8, no. 4, pp. 287-294, 2013.

[3] J. S. Sun, D. S. Goshi, and T. Itoh, "Optimization and modeling of sparse conformal retrodirective array", IEEE Trans. Antennas Propag., vol. 58, no. 3, pp. 977-981, Mar. 2010.

[4] T. N. Kaifas, and J. N. Sahalos, "On the Geometry Synthesis of Uniformy Excited Conformal Arrays by the Orthogonal Method," presented at the 2nd European Conf. on Antennas and Prop., 2007.

[5] G. Oliveri, E.T. Bekele, F. Robol, and A. Massa, "Sparsening Conformal Arrays Through a Versatile BCS-Based Method," IEEE Trans. Antennas Propag., vol. 62, no.4, pp. 1681-1689, Apr. 2014.

[6] G. Leone, "Source geometry optimization for hemispherical radiation pattern coverage," IEEE Trans. Antennas Propag., vol. 64, no. 5, pp .2033-2038, 2016.

[7] G. Leone, M.A. Maisto, and R. Pierri, "Application of inverse source reconstruction to conformal antennas synthesis," IEEE Trans. Antennas Propag., vol 66, no. 3, pp. 1436 - 1445, 2018.

[8] N. Tikhonov, and V. Y. Arsenin, Solution to ill-posed problems. Winston, 1977.

[9] M. Bertero, and P. Boccacci, Introduction to inverse problems in imaging. CRC press, 1998.

[10] G. Toraldo di Francia, "Degrees of freedom of an image," $J$. Opt. Soc. Am., vol. 59, no. 7, pp. 799-804, 1969.

[11] R. Piestun, and D. A. B. Miller, "Electromagnetic degrees of freedom of an optical system," J. Opt. Soc. Am. A, vol. 17, no. 5, pp. 892-902, 2000.

[12] G. Newsam, and R. Barakat, "Essential dimension as a welldefined number of degrees of freedom of finite-convolution operators appearing in optics," J. Opt. Soc. Am. A, vol. 2, no. 11, pp. 2040-2045, 1985.

[13] R. Pierri, A. Liseno, F. Soldovieri, and R. Solimene, "Indepth resolution for a strip source in the Fresnel zone," $J$. Opt. Soc. Am. A, vol. 18, no. 2, pp. 352-359, 2001.

[14] G. Leone, M.A. Maisto, and R. Pierri, "Inverse source of circumference geometries: SVD investigation based on Fourier analysis", Prog. Electromagn. Res. M, vol. 76, pp. 217-230, 2018.

[15] G. Leone, F. Munno, and R. Pierri, "Inverse source on conformal conic geometries," accepted for publication on IEEE Trans. Antennas Propag. .

[16] G. Leone, F. Munno, and R. Pierri "Synthesis of Angle Arrays by the NDF of the Radiation Integral," accepted for publication on IEEE Trans. Antennas Propag...

[17] C. Lanczos, Linear differential operators, vol. 18. SIAM, 1997. 